\title{
Lasiodiplodia chinensis, a new holomorphic species from China
}

\author{
Dou ZP', He W' $\mathbf{W h}^{\mathbf{1}}$ Zhang $\mathrm{Y}^{1}$ \\ ${ }^{1}$ Institute of Microbiology, PO Box 61, Beijing Forestry University, Beijing 100083, PR China \\ ${ }^{2}$ Beijing Key Laboratory for Forest Pest Control, Beijing Forestry University, Beijing 100083, PR China
}

Dou ZP, He W, Zhang Y 2017 - Lasiodiplodia chinensis, a new holomorphic species from China. Mycosphere 8(2), 521-532, Doi 10.5943/mycosphere/8/2/3.

\begin{abstract}
A new species of Lasiodiplodia (L. chinensis) is described and illustrated from several hosts collected from Hainan and Shandong Province in China. Both sexual and asexual states of $L$. chinensis were observed, which is characterized by its broadly clavate to clavate asci, fusiform, hyaline and aseptate ascospores, and initially hyaline, aseptate, ovoid to ellipsoid conidia that become pigmented and 1-2-septate with longitudinal striations when mature. Phylogenetically, $L$. chinensis is closely related to L. pseudotheobromae, L. sterculiae and L. lignicola. Morphological comparisons of these four species lead to the conclusion that the collected taxon is new to science
\end{abstract}

Key words - Botryosphaeriaceae - phylogeny - sexual morph - taxonomy

\section{Introduction}

Lasiodiplodia was formally introduced by Clendenin, (1896), and typified by L. theobromae (Phillips et al. 2013). Species of Lasiodiplodia are mostly distributed in tropical and subtropical regions where they can cause cankers, die-back, fruit or root rot, branch blight or discoloration on a wide range of woody hosts (Punithalingam 1980, Ismail et al. 2012, Phillips et al. 2013, Cruywagen et al. 2016). Forty-two species have been included in Lasiodiplodia (http://www.mycobank.org, Oct. 2016), of which thirty species have been described since 2004 (Phillips et al. 2013, Machado et al. 2014, Netto et al. 2014). Presently 31 species are known from culture (Dissanakake et al. 2016). However, the sexual morphs of Lasiodiplodia species have rarely been recorded and are known for only four Lasiodiplodia species, namely, L. gonubiensis Pavlic, Slippers \& M.J. Wingf., L. lignicola (Ariyaw., J.K. Liu \& K.D Hyde) A.J.L. Phillips, A. Alves \& Abdollahz, L. theobromae (Pat.) Griffon \& Maubl. and L. pseudotheobromae A.J.L. Phillips, A. Alves \& Crous (Liu et al. 2012, Phillips et al. 2013, Trakunyingcharoen et al. 2015, Tennakoon et al. 2016).

Lasiodiplodia pseudotheobromae was introduced by Alves et al. (2008) as a cryptic species in L. theobromae (Pat.) Griffon \& Maubl, which is a cosmopolitan pathogen that occurs on a large number of hosts in the tropics and subtropics (Alves et al. 2008, Machado et al. 2014, Wei et al. 2014, Dissanayake et al. 2015, Awan et al. 2016). In the course of an ongoing survey of Botryosphaeriaceae in China, a new species of Lasiodiplodia was obtained from woody plants in Hainan and Shandong Province. This species is closely related to but differs from $L$. pseudotheobromae. In this paper, we name and describe this species based on its sexual and asexual morphology, and resolve its phylogenetic position within Lasiodiplodia. 


\section{Materials \& Methods}

\section{Isolates and morphology}

Twigs were air-dried at room temperature and examined with an Olympus SZ 61 dissecting microscope without prior incubation in a moist chamber. Photomicrographs were taken with a Nikon Coolpix 995 digital camera fitted with an eyepiece adapter to the dissecting microscope. Microscopic observations of ascostromatal contents were carried out from material mounted in water. Thin vertical free-hand sections were made with a razor blade under the dissecting microscope and mounted in water. Photomicrographs were taken with a Nikon Coolpix 995 digital camera connected to a trinocular Leitz Orthoplan microscope and processed with Adobe Photoshop Elements 10 software. Measurements of ascospores, asci, hamathecial elements, conidia, paraphyses and conidiogenous cells were made from water mounts.

Isolations were made from ascomata or conidiomata on dead or dying twigs of different hosts and grown on $2 \%$ water agar (WA) (Biolab, S.A.), and subsequently transferred to synthetic nutrientpoor agar (SNA) with sterilized pine needles. Isolates grown on malt extract agar (MEA, Biolab, S.A.) were kept at ambient temperatures (about $26-28{ }^{\circ} \mathrm{C}$ ) in the dark to establish colony characteristics. Fungal isolates and herbarium specimens were deposited at Beijing Forestry University (BJFU) with duplicates in the China General Microbiological Culture Collection Center (CGMCC) and the Mycological Herbarium of the Institute of Microbiology, Chinese Academy of Sciences (HMAS).

\section{DNA extraction, PCR amplification}

Mycelium was grown on MEA plates and DNA extracted with CTAB plant genome DNA fast extraction kit (Aidlab Biotechnologies Co., Ltd, Beijing, China). The internal transcribed spacer of rDNA (ITS) was amplified and sequenced with primers ITS-1 and ITS-4 (White et al. 1990). The translation elongation factor- $1 \alpha$ (tefl- $\alpha)$ was amplified and sequenced with primers EF1-688F and EF1-1251R (Alves et al. 2008). The $\beta$-tubulin gene (TUB) was amplified and sequenced with primers Bt2a and Bt2b (Glass \& Donaldson 1995). The RPB2 sequences were amplified and sequenced using primers RPB2-LasF and RPB2-LasR (Cruywagen et al. 2016). PCR amplification and sequencing followed the protocol of Zhang et al. (2009).

\section{Sequence alignment and phylogenetic analysis}

The combined loci of ITS, RPB2, tefl- $\alpha$ and TUB were used to infer the phylogenetic relationships of the new species and another species of Lasiodiplodia. Sequences generated were analyzed with other sequences obtained from GenBank (Table 1). Alignments were conducted in MEGA v. 6 (Tamura et al. 2013) and phylogenetic analyses performed in PAUP v. 4.0b10 (Swofford 2002) and MrBayes v. 3.1.2 (Ronquist \& Huelsenbeck 2003). Prior to phylogenetic analysis, ambiguous sequences at the start and end were deleted and gaps manually adjusted to optimize the alignments. Maximum Parsimony (MP) was used to conduct heuristic searches as implemented in PAUP with the default options method (Zhang et al. 2008). Analyses were done under different parameters of maximum parsimony criteria as outlined in Zhang et al. (2008). Clade stability was assessed in a bootstrap analysis with 1000 replicates, random sequence additions with maxtrees set to 1000 and other default parameters as implemented in PAUP. For the MrBayes analysis, the best-fit model of nucleotide evolution $(\mathrm{GTR}+\mathrm{I}+\mathrm{G})$ was selected by Akaike information criterion (AIC; Posada \& Buckley 2004) in MrModeltest v. 2.3. The metropoliscoupled Markov Chain Monte Carlo (MCMCMC) approach was used to calculate posterior probabilities (Huelsenbeck \& Ronquist 2005). A preliminary Bayesian inference (BI) analysis using MrBayes software revealed that the Markov Chain Monte Carlo (MCMC; Huelsenbeck \& Ronquist, 2001) steady state was reached after less than 10,000 generations (the average standard deviation of split frequencies was constantly below 0.01). A conservative burn-in of 100 trees was chosen and a full analysis of 10,000,000 generations was carried out with sampling every 100 
generations. Trees were viewed in TREEVIEW. The nucleotide sequences generated in this paper were deposited in GenBank (Table 1). Trees and alignments were deposited in TreeBase (S20597).

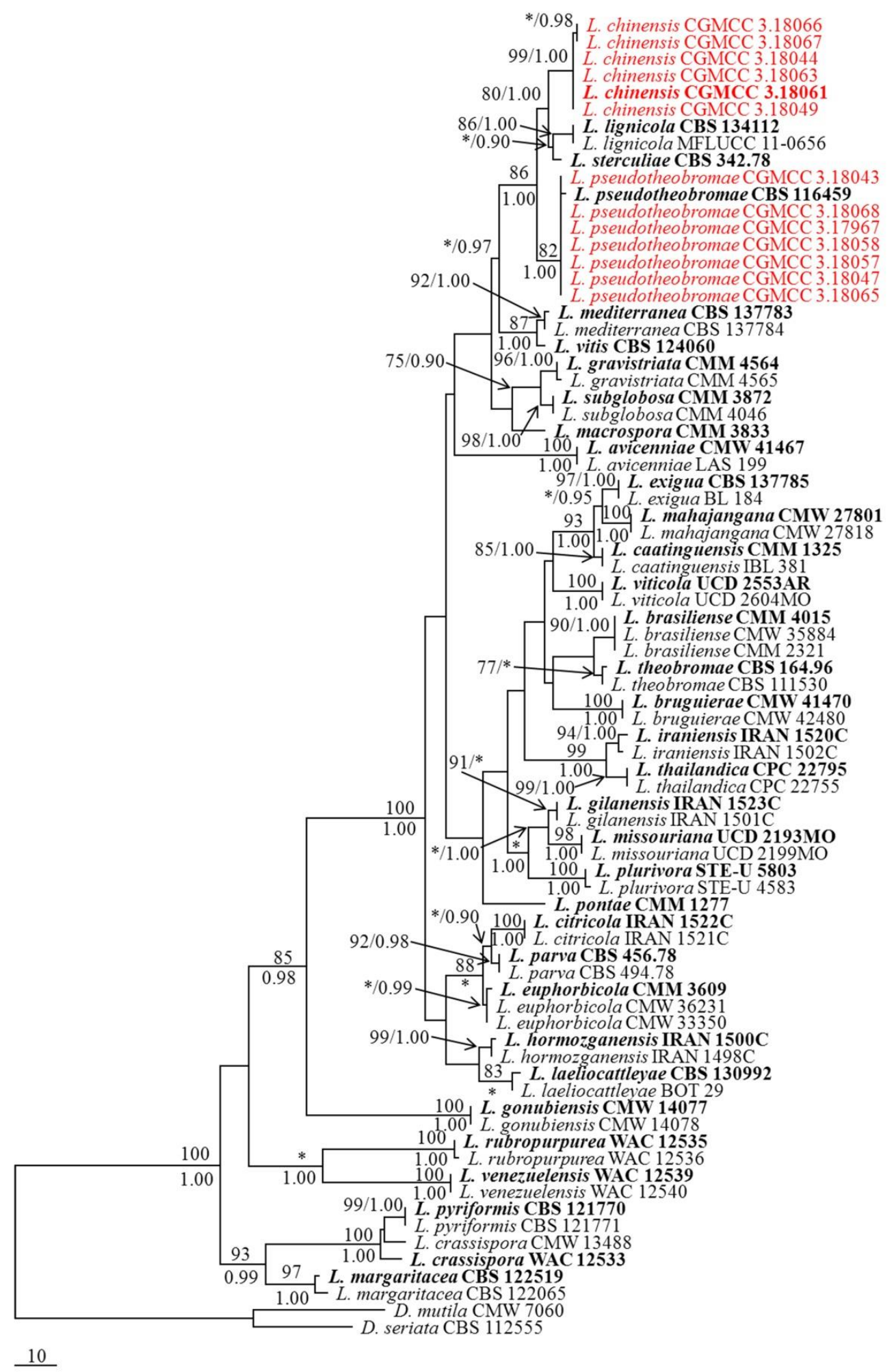

Fig. 1 - Maximum parsimony tree generated from sequence analysis of the combined ITS, tef1- $\alpha$, TUB and RPB2 dataset. Designated out group taxon is Diplodia mutila and D. seriata. Bootstrap support values for maximum parsimony (MP) greater than $75 \%$ are shown above the nodes, and Bayesian bootstrap (BP) support values above 0.90 are shown under the branches $(*=$ MP value less than $75 \%$ or BP value less than 0.90 ). The species characterized in this study are in red, and the ex-type strains are in boldface. 


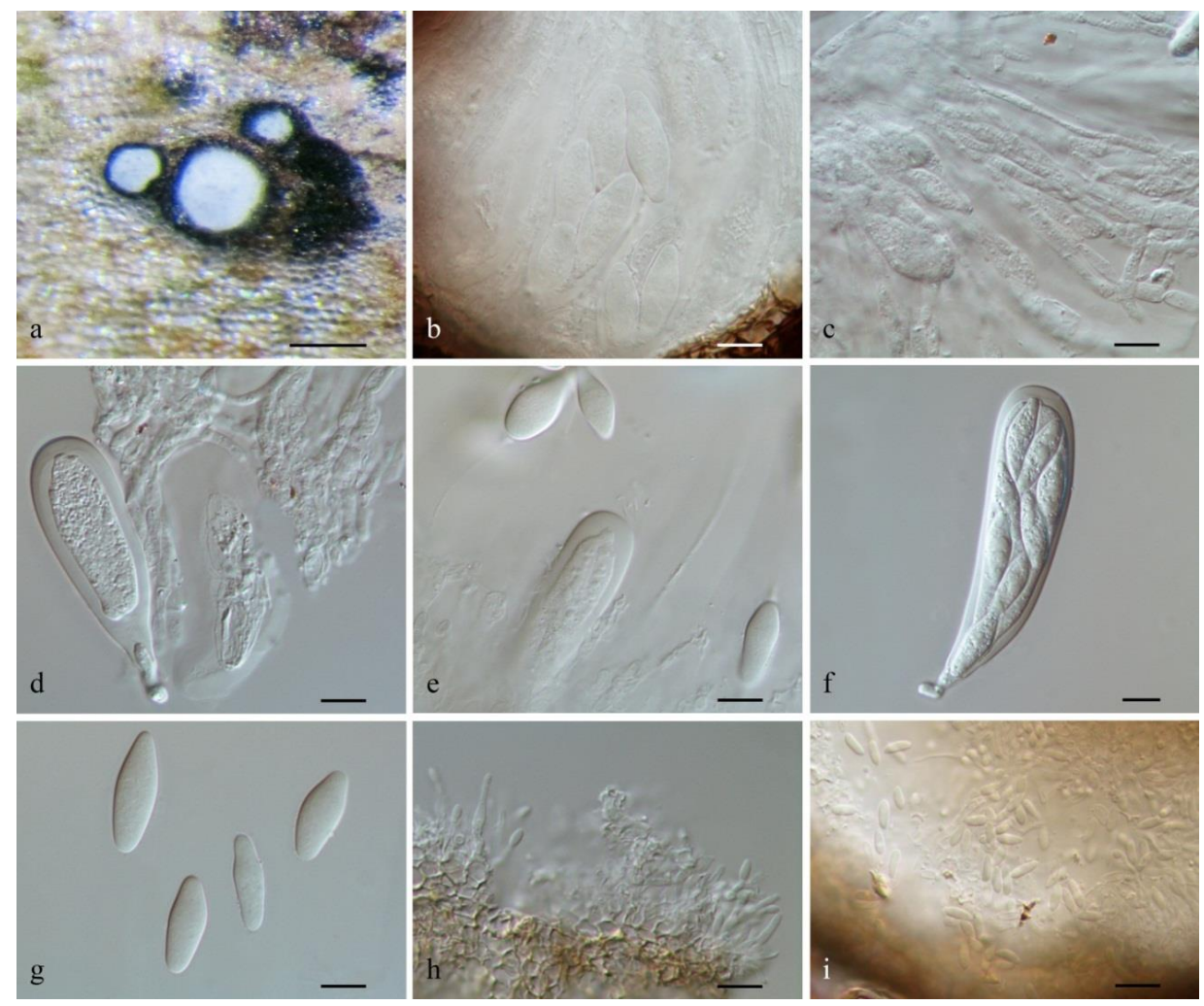

Fig. 2 - Lasiodiplodia chinensis (from holotype: HMAS247143) a Immersed ascomata on the host. b A section of ascoma with asci inside. c, d Cellular pseudoparaphyses and immature asci. e Ascus tip showing apical chamber and released ascospores. f A mature ascus. g Released ascospores. $h$ Spermatogenous cells. $i$ A section with spermatia inside. Scale bars: $a=200 \mu \mathrm{m}, b-i=10 \mu \mathrm{m}$.

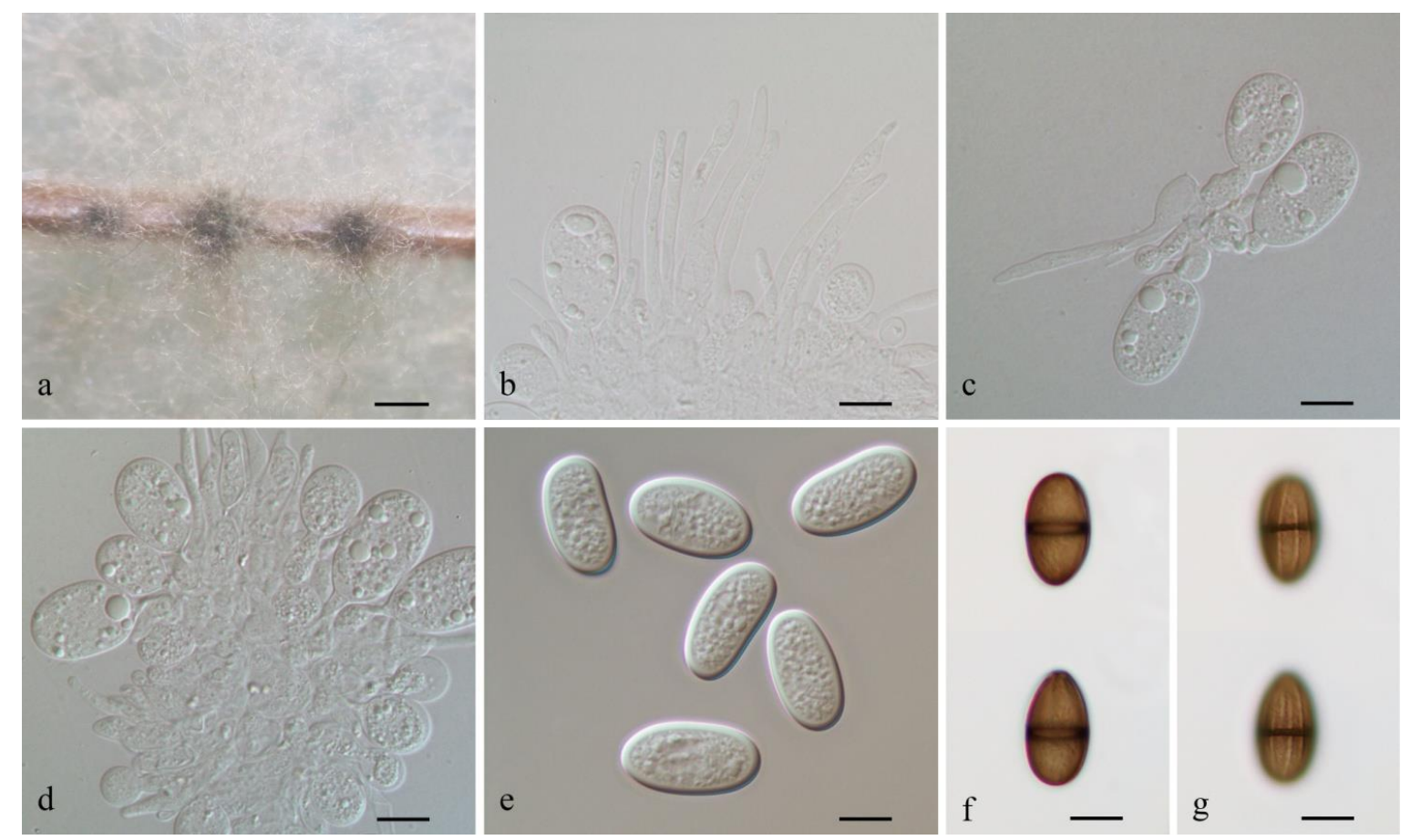

Fig. 3 - Lasiodiplodia chinensis (From ex-type: CGMCC3.18061) a. Conidiomata formed on pine needles in culture. $\mathrm{b}-\mathrm{d}$. Conidia developing on conidiogenous cells among paraphyses. e. Hyaline, immature conidia with granular content. $\mathrm{f}-\mathrm{g}$. Mature conidia in two different focal planes to show the longitudinal striations. Scale bars: $\mathrm{a}=500 \mu \mathrm{m} ; \mathrm{b}-\mathrm{g}=10 \mu \mathrm{m}$. 
Table 1 GenBank and culture collection accession numbers of species included in the phylogenetic study. Newly deposited sequences are shown in bold.

\begin{tabular}{|c|c|c|c|c|c|c|c|}
\hline \multirow[t]{2}{*}{ Species } & \multirow[t]{2}{*}{ Cultures } & \multirow[t]{2}{*}{ Host } & \multirow[t]{2}{*}{ Locality } & \multicolumn{4}{|c|}{ GenBank } \\
\hline & & & & ITS & tef1-a & TUB & RPB2 \\
\hline L. avicenniae & CMW 41467 & Avicennia marina & South Africa & KP860835 & KP860680 & KP860758 & KU587878 \\
\hline L. avicenniae & LAS 199 & Avicennia marina & South Africa & KU587957 & KU587947 & KU587868 & KU587880 \\
\hline L. brasiliense & CMM 4015 & Mangifera indica & Brazil & JX464063 & JX464049 & N/A & $\mathrm{N} / \mathrm{A}$ \\
\hline L. brasiliense & CMM 2321 & Carica papaya & Brazil & KC484797 & KC481528 & N/A & N/A \\
\hline L. brasiliense & CMW 35884 & Adansonia madagascariensis & Madagascar & KU887094 & KU886972 & KU887466 & KU696345 \\
\hline L. bruguierae & CMW 41470 & Bruguiera gymnorrhiza & South Africa & KP860833 & KP860678 & KP860756 & KU587875 \\
\hline L. bruguierae & CMW 42480 & Bruguiera gymnorrhiza & South Africa & KP860832 & KP860677 & KP860755 & KU587876 \\
\hline L. caatinguensis & CMM 1325 & Citrus sinensis & Brazil & KT154760 & КT008006 & KT154767 & N/A \\
\hline L. caatinguensis & IBL 381 & Spondias purpurea & Brazil & KT154757 & KT154751 & KT154764 & N/A \\
\hline L. chinensis & CGMCC3.18061 & unknown & China & KX499889 & KX499927 & KX500002 & KX499965 \\
\hline L. chinensis & CGMCC3.18044 & Vaccinium uliginosum & China & KX499875 & KX499913 & KX499988 & KX499951 \\
\hline L. chinensis & CGMCC3.18049 & Rhodomyrtus tomentosa & China & KX499878 & KX499916 & KX499991 & KX499954 \\
\hline L. chinensis & CGMCC3.18063 & Canarium parvum & China & KX499891 & KX499929 & KX500004 & KX499967 \\
\hline L. chinensis & CGMCC3.18066 & Hevea brasiliensis & China & KX499899 & KX499937 & KX500012 & KX499974 \\
\hline L. chinensis & CGMCC3.18067 & Sterculia lychnophora & China & KX499901 & KX499939 & KX500014 & KX499976 \\
\hline L. citricola & IRAN 1522C & Citrus sp. & Iran & GU945354 & GU945340 & KU887505 & KU696351 \\
\hline L. citricola & IRAN 1521C & Citrus sp. & Iran & GU945353 & GU945339 & KU887504 & KU696350 \\
\hline L. crassispora & WAC 12533 & Santalum album & Australia & DQ103550 & DQ103557 & KU887506 & KU696353 \\
\hline L. crassispora & CMW 13488 & Eucalyptus urophylla & Venezuela & DQ103552 & DQ103559 & KU887507 & KU696352 \\
\hline L. euphorbiicola & CMM 3609 & Jatropha curcas & Brazil & KF234543 & KF226689 & KF254926 & N/A \\
\hline L. euphorbiicola & CMW 33350 & Adansonia digitata & Botswana & KU887149 & KU887026 & KU887455 & KU696346 \\
\hline L. euphorbiicola & CMW 36231 & Adansonia digitata & Zimbabwe & KU887187 & KU887063 & KU887494 & KU696347 \\
\hline L. exigua & CBS 137785 & Retama raetam & Tunisia & KJ638317 & KJ638336 & KU887509 & KU696355 \\
\hline L. exigua & BL 184 & Retama raetam & Tunisia & KJ638318 & KJ638337 & N/A & N/A \\
\hline L. gilanensis & IRAN $1523 \mathrm{C}$ & Unknown & Iran & GU945351 & GU945342 & KU887511 & KU696357 \\
\hline L. gilanensis & IRAN 1501C & Unknown & Iran & GU945352 & GU945341 & KU887510 & KU696356 \\
\hline L. gonubiensis & CMW 14077 & Syzygium cordatum & South Africa & AY639595 & DQ103566 & DQ458860 & KU696359 \\
\hline L. gonubiensis & CMW 14078 & Syzygium cordatum & South Africa & AY639594 & DQ103567 & EU673126 & KU696358 \\
\hline
\end{tabular}


Table 1 (cont.)

\begin{tabular}{|c|c|c|c|c|c|c|c|}
\hline \multirow[t]{2}{*}{ Species } & \multirow[t]{2}{*}{ Cultures } & \multirow[t]{2}{*}{ Host } & \multirow[t]{2}{*}{ Locality } & \multicolumn{4}{|c|}{ GenBank } \\
\hline & & & & ITS & tef1-a & TUB & RPB2 \\
\hline L. gravistriata & CMM 4564 & Anacardium humile & Brazil & KT250949 & KT250950 & N/A & N/A \\
\hline L. gravistriata & CMM 4565 & Anacardium humile & Brazil & KT250947 & KT266812 & N/A & N/A \\
\hline L. hormozganensis & IRAN 1500C & Olea sp. & Iran & GU945355 & GU945343 & KU887515 & KU696361 \\
\hline L. hormozganensis & IRAN 1498C & Mangifera indica & Iran & GU945356 & GU945344 & KU887514 & KU696360 \\
\hline L. iraniensis & IRAN $1520 \mathrm{C}$ & Salvadora persica & Iran & GU945348 & GU945336 & KU887516 & KU696363 \\
\hline L. iraniensis & IRAN 1502C & Juglans sp. & Iran & GU945347 & GU945335 & KU887517 & KU696362 \\
\hline L. laeliocattleyae & CBS 130992 & Mangifera indica & Egypt & JN814397 & JN814424 & KU887508 & KU696354 \\
\hline L. laeliocattleyae & BOT 29 & Mangifera indica & Egypt & JN814401 & JN814428 & N/A & N/A \\
\hline L. lignicola & CBS 134112 & dead wood & Thailand & JX646797 & KU887003 & JX646845 & KU696364 \\
\hline L. lignicola & MFLUCC 11-0656 & dead wood & Thailand & JX646798 & N/A & JX646846 & N/A \\
\hline L. macrospora & CMM 3833 & Jatropha curcas & Brazil & KF234557 & KF226718 & KF254941 & N/A \\
\hline L. mahajangana & CMW 27801 & Terminalia catappa & Madagascar & FJ900595 & FJ900641 & FJ900630 & KU696365 \\
\hline L. mahajangana & CMW 27818 & Terminalia catappa & Madagascar & FJ900596 & FJ900642 & FJ900631 & KU696366 \\
\hline L. margaritacea & CBS 122519 & Adansonia gibbosa & Western Australia & EU144050 & EU144065 & KU887520 & KU696367 \\
\hline L. margaritacea & CBS 122065 & Adansonia gibbosa & Western Australia & EU144051 & EU144066 & N/A & N/A \\
\hline L. mediterranea & CBS 137783 & Quercus ilex & Italy & KJ638312 & KJ638331 & KU887521 & KU696368 \\
\hline L. mediterranea & CBS 137784 & Vitis vinifera & Italy & KJ638311 & KJ638330 & KU887522 & KU696369 \\
\hline L. missouriana & UCD $2193 \mathrm{MO}$ & Vitis sp. & USA & HQ288225 & HQ288267 & HQ288304 & KU696370 \\
\hline L. missouriana & UCD 2199MO & Vitis sp. & USA & HQ288226 & HQ288268 & HQ288305 & KU696371 \\
\hline L. parva & CBS 456.78 & Cassava field-soil & Colombia & EF622083 & EF622063 & KU887523 & KU696372 \\
\hline L. parva & CBS 494.78 & Cassava field-soil & Colombia & EF622084 & EF622064 & EU673114 & KU696373 \\
\hline L. plurivora & STE-U 5803 & Prunus salicina & South Africa & EF445362 & EF445395 & KU887524 & KU696374 \\
\hline L. plurivora & STE-U 4583 & Vitis vinifera & South Africa & AY343482 & EF445396 & KU887525 & KU696375 \\
\hline L. pontae & CMM 1277 & Spondias purpurea & Brazil & KT151794 & KT151791 & KT151797 & N/A \\
\hline L. pseudotheobromae & CBS 116459 & Gmelina arborea & Costa Rica & EF622077 & EF622057 & EU673111 & KU696376 \\
\hline L. pseudotheobromae & CGMCC3.18043 & Morus alba Linn & China & KX499872 & KX499910 & KX499985 & KX499948 \\
\hline L. pseudotheobromae & CGMCC3.18047 & Pteridium aquilinum & China & KX499876 & KX499914 & KX499989 & KX499952 \\
\hline L. pseudotheobromae & CGMCC3.18057 & Lagerstroemia indica & China & KX499885 & KX499923 & KX499998 & KX499961 \\
\hline
\end{tabular}




\begin{tabular}{|c|c|c|c|c|c|c|c|}
\hline \multirow[t]{2}{*}{ Species } & \multirow[t]{2}{*}{ Cultures } & \multirow[t]{2}{*}{ Host } & \multirow[t]{2}{*}{ Locality } & \multicolumn{4}{|c|}{ GenBank } \\
\hline & & & & ITS & tef1-a & TUB & RPB2 \\
\hline L. pseudotheobromae & CGMCC3.18058 & unknown & China & KX499887 & KX499925 & KX500000 & KX499963 \\
\hline L. pseudotheobromae & CGMCC3.18065 & unknown & China & KX499895 & KX499933 & KX500008 & N/A \\
\hline L. pseudotheobromae & CGMCC3.17967 & unknown & China & KX499897 & KX499935 & KX500010 & KX499972 \\
\hline L. pseudotheobromae & CGMCC3.18068 & unknown & China & KX499902 & KX499940 & KX500015 & KX499977 \\
\hline L. pyriformis & CBS 121770 & Acacia mellifera & Namibia & EU101307 & EU101352 & KU887527 & KU696378 \\
\hline L. pyriformis & CBS 121771 & Acacia mellifera & Namibia & EU101308 & EU101353 & KU887528 & KU696379 \\
\hline L. rubropurpurea & WAC 12535 & Eucalyptus grandis & Australia & DQ103553 & DQ103571 & EU673136 & KU696380 \\
\hline L. rubropurpurea & WAC 12536 & Eucalyptus grandis & Australia & DQ103554 & DQ103572 & KU887530 & KU696381 \\
\hline L. sterculiae & CBS 342.78 & Sterculia oblonga & Germany & KX464140 & KX464634 & KX464908 & KX463989 \\
\hline L. subglobosa & CMM 3872 & Jatropha curcas & Brazil & KF234558 & KF226721 & KF254942 & N/A \\
\hline L. subglobosa & CMM 4046 & Jatropha curcas & Brazil & KF234560 & KF226723 & KF254944 & N/A \\
\hline L. thailandica & CPC 22795 & Mangifera indica & Thailand & KJ193637 & KJ193681 & N/A & N/A \\
\hline L. thailandica & CPC 22755 & Phyllanthus acidus & Thailand & KM006433 & KM006464 & N/A & N/A \\
\hline L. theobromae & CBS 164.96 & Fruit along coral reef coast & Papua New Guinea & AY640255 & AY640258 & KU887532 & KU696383 \\
\hline L. theobromae & CBS 111530 & Unknown & Unknown & EF622074 & EF622054 & KU887531 & KU696382 \\
\hline L. venezuelensis & WAC 12539 & Acacia mangium & Venezuela & DQ103547 & DQ103568 & KU887533 & KU696384 \\
\hline L. venezuelensis & WAC 12540 & Acacia mangium & Venezuela & DQ103548 & DQ103569 & KU887534 & N/A \\
\hline L. viticola & UCD 2553AR & Vitis sp. & USA & HQ288227 & HQ288269 & HQ288306 & KU696385 \\
\hline L. viticola & UCD 2604MO & Vitis sp. & USA & HQ288228 & HQ288270 & HQ288307 & KU696386 \\
\hline L. vitis & CBS 124060 & Vitis vinifera & Italy & KX464148 & KX464642 & KX464917 & KX463994 \\
\hline Diplodia mutila & CMW 7060 & Fraxinus excelsior & Netherlands & AY236955 & AY236904 & AY236933 & EU339574 \\
\hline D. seriata & CBS 112555 & Vitis vinifera & Portugal & AY259094 & AY573220 & DQ458856 & N/A \\
\hline
\end{tabular}




\section{Results}

\section{Phylogenetic analyses}

Phylogenetic analysis of the combined ITS, tef1- $\alpha$, TUB and RPB2 sequence dataset comprising $1957 \mathrm{bp}$ revealed 301 parsimony-informative characters. The outgroup taxa were Diplodia mutila and $D$. seriata. The heuristic search with random addition of taxa (1,000 replicates) generated 504 most parsimonious trees of 762 steps $(\mathrm{CI}=0.646, \mathrm{RI}=0.866, \mathrm{RC}=0.559, \mathrm{HI}=$ 0.354). In the phylogenetic tree, the clade comprising L. chinensis, L. lignicola, L. sterculiae and $L$. pseudotheobromae as well as the subclades comprising individual species of $L$. chinensis and $L$. pseudotheobromae all received high bootstrap support (Fig. 1). The clade comprising L. chinensis, L. sterculiae and L. lignicola also received high support for Bayesian analysis, while moderate support for MP analysis (Fig. 1).

\section{Taxonomy}

Lasiodiplodia chinensis Z. P. Dou, Y. Zhang ter., sp. nov.

Figs 2, 3

MycoBank 819527; Facesoffungi number: FoF02831

Etymology - The epithet chinensis refers to China, the country from which it is described.

Saprobic or pathogen, associated with woody branches. Sexual morph: Ascomata immersed under bark, sometimes erumpent, globose to subglobose, solitary or in small groups of up to 4, black, $140-290 \mu \mathrm{m}$ diam., $170-185 \mu \mathrm{m}$ high. Papilla up to $28-43 \mu \mathrm{m}$ diam. wide, with a central ostile. Peridium 12-49 $\mu \mathrm{m}$ wide, thin to thick-walled, composed of several layers of dark brown to black cells of textura angularis. Pseudoparaphyses hyaline, cellular, septate, constricted at the septum, 3-4 $\mu \mathrm{m}$ wide. Asci bitunicate with thick endotunica and well-developed apical chamber best seen in water, with a short, furcate pedicel, broadly clavate to clavate, 53-90(-116) × 14-23(25) $\mu \mathrm{m}, 8$-spored. Ascospores overlapping bi- to tri-seriate, fusiform to broadly fusiform, with rounded ends, the upper half often broader than the lower half, hyaline, aseptate, straight or slightly curved, smooth-walled, 19-25 × 6-9 $\mu \mathrm{m}$ (av. of 30 conidia $=22.1 \times 7.6 \mu \mathrm{m})$. Asexual morph: Conidiomata stromatic, produced on pine needles on SNA within 1-2 wk, semi-immersed, sometimes superficial, solitary, papillate, uniloculate, dark brown to black, covered with dense mycelium, 210-320 $\mu \mathrm{m}$ diam. Paraphyses cylindrical, initially aseptate, becoming up to 9-septate when mature, unbranched, occasionally basal cells swollen, hyaline, up to $99 \mu \mathrm{m}$ long, 3-7 $\mu \mathrm{m}$ wide. Conidiophores reduced to conidiogenous cells. Conidiogenous cells holoblastic, hyaline, cylindrical to ampulliform, proliferating percurrently, $(8-) 10-15(-18) \times 4-6(-7) \mu \mathrm{m}(\mathrm{av} .=12.4 \times$ $5.0 \mu \mathrm{m}, \mathrm{n}=20$ ). Conidia produced in culture initially hyaline, unicellular, ovoid to ellipsoid, thickwalled with granular content, round at apex, occasionally truncate at base, becoming pigmented while did not attach to the conidiogenous cell, with one septum when mature, developing longitudinal striations when mature, $(18-) 19-25 \times 12-14 \mu \mathrm{m}$ (av. of 30 conidia $=21.9 \times 12.6 \mu \mathrm{m}$, $\mathrm{L} / \mathrm{W}$ ratio $=1.75$, range from 1.43 to 2.08 ). Spermatogenous cells discrete or integrated, hyaline, smooth, fusiform to broadly cylindrical to ampulliform, holoblastic, proliferating percurrently or proliferating internally resulting in annellations, $8-15 \times 2-3 \mu \mathrm{m}$. Spermatia hyaline, smooth, aseptate, ellipsoidal to allantoid with rounded ends, $5-7 \times 2 \mu \mathrm{m}$.

Culture characteristics - Colonies on MEA at $28{ }^{\circ} \mathrm{C}$ in darkness, initially white with dense aerial mycelia, becoming pale olivaceous grey to olivaceous grey on the surface within $10 \mathrm{~d}$, with the reverse sides of the colonies olivaceous grey to olivaceous black. Colonies reaching an average of $79 \mathrm{~mm}$ on MEA after $2 \mathrm{~d}$ in the dark at $28^{\circ} \mathrm{C}$.

Specimens examined - CHINA, Hainan Province, Wanning City, Xinglong medical plants garden, from branch of an unknown woody plant, 4 November 2015, W. He \& Z.P. Dou (HMAS247143, holotype), ex-type living culture, CGMCC3.18061; Haikou City, Meilan District, from branch of Canarium parvum, 6 November 2015, Y. Zhang \& Y.P. Zhou (CGMCC3.18063); from branch of Hevea brasiliensis, 6 November 2015, Y. Zhang \& Y.P. Zhou (CGMCC3.18066); Danzhou City, the Danzhou Tropical Botanical Garden, from branch of Rhodomyrtus tomentosa, 3 
November 2015, Y. Zhang \& Y.P. Zhou (CGMCC3.18049); from branch of Sterculia lychnophora, 3 November 2015, W. He \& Z.P. Dou (HMAS247144, CGMCC3.18067); Shandong province, Qingdao City, Huangdao District, from branch of Vaccinium uliginosum, 13 December 2014, J.H. Zhao (Paratype, HMAS247145, CGMCC3.18044).

Notes - Phylogenetic analyses based on combined ITS, RPB2, TUB and tefl-a sequences indicated that L. chinensis is closely related to L. pseudotheobromae, L. sterculiae and L. lignicola, but the golden to dark brown mature ascospores of L. pseudotheobromae and L. lignicola differentiate these two species from L. chinensis (Tennakoon et al. 2016). Only the asexual morph has been reported for $L$. sterculiae, which differs from L. chinensis by its hyaline and smaller conidia $((12-) 14-16(-17) \times(8-) 10-11(-12) \mu \mathrm{m})$ (Yang et al 2016).

\section{Discussion}

Of the five species of Lasiodiplodia with known sexual morphs, namely L. chinensis, $L$. pseudotheobromae, L. lignicola, L. gonubiensis and L. theobromae, golden to dark brown ascospores have been reported for L. pseudotheobromae and L. lignicola (Liu et al. 2012, Tennakoon et al. 2016). The ascospores of L. gonubiensis are initially hyaline, and then turn pale brown, 1-2-septate within ascoma or shortly after discharge (Trakunyingcharoen et al. 2015). No pigmented or septate ascospores of $L$. chinensis were observed in the present study. Although $L$. theobromae have been reported to have hyaline ascospores, the connection between L. theobromae and its sexual morph has not been proven conclusively (Phillips et al. 2013). However, $L$. theobromae is phylogenetically separated from L. chinensis (Fig. 3). So far, the sexual morphs of Lasiodiplodia species can be defined as having hyaline to dark brown aseptate ascospores, which can develop one or two septa when aged. Interestingly, of the five species with sexual stage reported, four of the reports are from Asia, i.e. L. pseudotheobromae and L. chinensis from China (Tennakoon et al. 2016, this study), L. lignicola and L. gonubiensis from Thailand (Liu et al. 2012, Trakunyingcharoen et al. 2015). Six collections of Lasiodiplodia chinensis have been obtained from both tropical (Hainan Province) and temperate regions (Shandong Province) in China, and the hosts include Canarium parvum (Burseraceae), Hevea brasiliensis (Euphorbiaceae), Rhodomyrtus tomentosa (Myrtaceae), Sterculia lychnophora (Malvaceae) and Vaccinium uliginosum (Vaccinieae). This indicates the wide geographic distribution and broad host spectrum of $L$. chinensis.

\section{Acknowledgement}

This study was financially supported by NSFC Projects of National Natural Science Foundation of China (General Program, 31370063), International Cooperation and Exchanges (31461143028) and National Science and Technology Foundation Project (2014FY210400). Prof. Dr. Chen Liang (Qingdao Agriculture University) is acknowledged for providing us the fungal specimens and isolates from Shandong Province.

\section{References}

Awan QN, Akgül DS, Unal G. 2016 - First Report of Lasiodiplodia pseudotheobromae Causing Postharvest Fruit Rot of Lemon in Turkey. Plant Disease 100, 2327.

Alves A, Crous PW, Correia A, Phillips AJL. 2008 - Morphological and molecular data reveal cryptic species in Lasiodiplodia theobromae. Fungal Diversity 28, 1-13.

Clendenin I. 1896 - Lasiodiplodia Ellis \& Everh. n. gen. Botanical Gazette Crawfordsville 21, $92-$ 93.

Dissanayake AJ, Zhang W, Mei L, Chukeatirote E et al. 2015 - Lasiodiplodia pseudotheobromae causes pedicel and peduncle discolouration of grapes in China. Australasian Plant Disease Notes 10, 1-5.

Dissanayake AJ, Phillips AJL, Li XH, Hyde KD 2016 - Botryosphaeriaceae: Current status of genera and species. Mycosphere 7(7), 1001-1073. 
Glass NL, Donaldson GC. 1995 - Development of primer sets designed for use with the PCR to amplify conserved genes from filamentous Ascomycetes. Applied \& Environmental Microbiology 61, 1323-1330.

Huelsenbeck JP, Ronquist F. 2001 - MRBAYES: Bayesian inference of phylogenetic trees. Bioinformatics 17, 754-755.

Ismail AM, Cirvilleri G, Polizzi G, Crous PW et al. 2012 - Lasiodiplodia species associated with dieback disease of mango (Mangifera indica) in Egypt. Australasian Plant Pathology 41: 649-660.

Liu JK, Phookamsak R, Doilom M, Wikee S et al. 2012 - Towards a natural classification of Botryosphaeriales. Fungal Diversity 57, 149-210.

Machado AR, Pinho DB, Pereira OL. 2014 - Phylogeny, identification and pathogenicity of the Botryosphaeriaceae associated with collar and root rot of the biofuel plant Jatropha curcas in Brazil, with a description of new species of Lasiodiplodia. Fungal Diversity 67, 1-17.

Netto MSB, Assunção IP, Lima GSA, Marques MW et al. 2014 - Species of Lasiodiplodia associated with papaya stem-end rot in Brazil. Fungal Diversity 67, 127-41.

Phillips AJL, Alves A, Abdollahzadeh J, Slippers B et al. 2013 - The Botryosphaeriaceae: genera and species known from culture. Studies in Mycology 76, 51-167.

Punithalingam E. 1980. Plant diseases attributed to Botryodiplodia theobromae Pat. Berlin: J. Cramer.

Ronquist F, Huelsenbeck JP. 2003 - MrBayes3: Bayesian phylogenetic inference under mixed models. Bioinformatics 19, 1572-1574.

Swofford DL. 2002. PAUP*. Phylogenetic Analysis Using Parsimony (*and Other Methods). Version 4. Sinauer Associates, Sunderland, Massachusetts.

Tamura K, Stecher G, Peterson D, Filipski A et al. 2013 - MEGA6: molecular evolutionary genetics analysis version 6.0. Molecular Biology \& Evolution 30, 2725-2729.

Tennakoon DS, Phillips AJL, Phookamsak R, Ariyawansa HA et al. 2016 - Sexual morph of Lasiodiplodia pseudotheobromae (Botryosphaeriaceae, Botryosphaeriales, Dothideomycetes) from China. Mycosphere 7, 990-1000.

Trakunyingcharoen T, Lombard L, Groenewald JZ, Cheewangkoon R et al. 2015 - Caulicolous Botryosphaeriales from Thailand. Persoonia 34, 87-99.

Wei W, Jiang N, Mei YN, Chu YL et al. 2014 - An antibacterial metabolite from Lasiodiplodia pseudotheobromae F2. Phytochemistry 100, 103-109.

White TJ, Bruns T, Lee S, Taylor J. 1990. Amplification and direct sequencing of fungal ribosomal RNA genes for phylogenetics. In: Innis MA, Gelfand DH, Sninsky JJ, White TJ (eds), PCR Protocols: a guide to methods and applications. Academic Press, New York, pp. 315-322.

Zhang Y, Jeewon R, Fournier J, Hyde KD. 2008 - Multi-gene phylogeny and morphotaxonomy of Amniculicola lignicola: a novel freshwater fungus from France and its relationships to the Pleosporales. Mycological Research 112, 1186-1194. 